\title{
Labyrinthe
}

36 | 2011 (1)

«Par les Grecs »

\section{Photo-, phono- et cinématographie chez Clément Rosset}

Pierre-Yves Macé

\section{(2) OpenEdition \\ 12 Journals}

Édition électronique

URL : http://journals.openedition.org/labyrinthe/4108

DOI : $10.4000 /$ labyrinthe.4108

ISSN : 1950-6031

Éditeur

Hermann

Édition imprimée

Date de publication : 15 février 2011

Pagination : 105-117

ISBN : 9782705680541

\section{Référence électronique}

Pierre-Yves Macé, «Photo-, phono- et cinématographie chez Clément Rosset », Labyrinthe [En ligne], 36 | 2011 (1), mis en ligne le 01 février 2013, consulté le 19 avril 2019. URL : http:// journals.openedition.org/labyrinthe/4108; DOI : 10.4000/labyrinthe.4108

Propriété intellectuelle 


\title{
Photo-, phono- et cinématographie chez Clément Rosset ${ }^{1}$
}

\author{
Pierre-Yves MACÉ \\ Contact: pymace@yahoo.fr
}

Condensée en une suite d'ouvrages très brefs, [l'œuvre] de Clément Rosset, opiniâtrement resserrée sur un seul concept (et ses dérivés), non des moindres il est vrai, celui de réel, ne se laisse pas appréhender facilement. Si elle se rend intelligible sans réserve au lecteur qu'elle réjouit par sa fraîcheur, son style alerte et son choix d'exemples associant Wittgenstein, Tati et Tintin, l'accès qu'elle offre au commentateur apparaît bien mince. Sa réfutation est bien souvent, sinon impossible, du moins improductive ou stérile, cependant que son approbation ne conduit qu'à sa réexpression tautologique - ce qui n'a rien d'étonnant considérant que l'approbation sans réserve de ce qui est en constitue le moteur tandis que la tautologie en est l'un des objets d'élection (cf. Le Démon de la Tautologie). En bref, le système-Rosset est à prendre ou à laisser, il s'importe ou se transpose difficilement dans une pensée qui essaierait de le questionner et le dépasser.

Aussi les lignes qui suivent ne discutent-elles les écrits de Rosset que lorsque ceux-ci abordent une question bien particulière qui à la fois déborde le champ de l'ontologie du réel et dans le même temps y renvoie sans cesse avec insistance : il s'agit du statut des photographies, phonographies et cinématographies, c'est-à-dire toutes les images (au sens étendu, visuel et/ou sonore) ou représentations qui fixent une matière sensible issue du réel par un moyen technique d'impression matérielle. Se revendiquant peu renseigné sur les questions relatives à la technique ${ }^{2}$,

1. Nous renverrons aux œuvres de Clément Rosset par les abréviations suivantes : RD (Le Réel et son double, Gallimard, 1976), TI (Le Réel, traité de l'idiotie, Minuit, 1977), OS (L'Objet singulier, Minuit, 1979), PC (Le Principe de cruauté, Minuit, 1988), DT (Le Démon de la tautologie, Minuit, 1997), IF (Impressions fugitives, Minuit, 2004), F (Fantasmagories, Minuit, 2006).

2. Dans un entretien accordé à Philosophie Magazine (n¹7, Paris, Philo Éditions, mars 2008, p. 56-61) Rosset déclare ne pas bien connaître le domaine mal nommé du « virtuel » au sujet duquel on l'interroge, et se démarque de Baudrillard précisément sur cette question de la technique: « un philosophe obnubilé par la technologie - dont je ne me suis jamais senti proche pour cette raison » (p. 58). 
Rosset consacre pourtant quelques chapitres de ses livres, voire un livre entier (Fantasmagories, 2005) à la photographie et/ou au cinéma, non tant pour analyser le détail de leurs productions que pour soumettre leur principe même (leur technique, donc) à la question qui est au cœur de tous ses écrits : quel est le rapport au réel entretenu par ces images?

Ces images sont problématiques en ce sens qu'elles questionnent le partage entre ce qui peut de bon droit être considéré comme réel et ce qui ne l'est pas - partition autour de laquelle toute l'œuvre de Rosset se meut. Le réel y obéit à un critère de détermination unique. Il est la forme de la singularité ou de l'idiotie, au sens premier, étymologique de ce terme: singularité, unicité, particularité ${ }^{3}$. Son mode d'expression est tautologique: $\mathrm{A}=\mathrm{A}$ (DT, p. 11). Exemple célèbre exposé dans L'Objet Singulier (1979): le camembert que je goûte et que j'échoue à déterminer autrement qu'en disant: « voilà du camembert » (OS, p. 22). Symétriquement l'irréel (ou encore l'illusoire) est le non-singulier, c'està-dire ce qui se présente à l'état de double. Est irréel l'événement ou l'objet venant redoubler ou se superposer à un événement ou objet du réel qui par définition - ontologiquement - ne peut être qu'un. Exemple: la façon dont Swann, le personnage proustien, dédouble la figure aimée d'Odette en deux composantes incompatibles : la femme aimée $e t$ la femme entretenue, afin de ne pas voir la réalité qui précisément les conjoint (la femme qu'il aime est une femme entretenue, soit $\mathrm{A}=\mathrm{A})^{4}$.

Toute représentation du réel est considérée comme sa négation car valant comme son «double». Bien avant donc d'être produite par des appareils fixant le réel sensible, l'image est, dans la généralité inclusive de son concept, depuis les peintures de Lascaux jusqu'aux images numériques d'aujourd'hui, placée du côté du non-réel. Cependant, à la différence de Platon, l'une des grandes cibles de la philosophie rossetienne, Rosset ne blâme pas les images de leur non-fidélité au réel. Sa critique porte sur la mimesis plutôt que sur les images en elles-mêmes: elle postule comme impossible la représentation du réel, (puisque celui-ci est précisément le singulier qui ne tolère aucune doublure), mais elle accepte en revanche

3. Dans le grec ancien $\square \delta i \omega ́ \tau\rceil \zeta$, idiôtês (dont le sens est voisin du moderne « idiot »), on peut déjà saisir la racine $\square \delta \iota_{0}$, idios, « qui appartient en propre », origine que l'on retrouve dans des mots comme idiome ou idiosyncrasie.

4. Cet exemple est commenté dans Le Réel et son double (RD, 17-18), puis repris dans Le Principe de cruauté (PC, 22-23). 


\section{Photo-, phono- et cinématographie chez Clément Rosset}

- voire encourage - une pratique de l'image que l'on pourrait dire autonome, qui s'éloigne résolument de la réalité perceptuelle 5 .

Chez Rosset, la distinction entre les différentes images se joue là : entre les images qui consentent à leur statut d'image et celles qui paraissent être davantage que de simples images, et qui, par leur proximité apparente au réel, semblent se mettre en concurrence directe avec lui. Tout occupées à reproduire mécaniquement le réel, ces images-là sont celles qui en relèvent le moins. Le philosophe livre ainsi, au sujet du cinéma, un diagnostic dont on appréciera le caractère paradoxal:

Si l'on entend par le réel tout ce qui se présente comme stricte singularité - et est par conséquent hors d'état d'être jamais re-présenté - on est tenté d'estimer de prime abord que, de tous les arts, le cinéma est celui qui entretient le moins de rapport avec la réalité: pour appartenir essentiellement non au domaine du réel, mais à l'univers de ses doubles. (OS, p. 51)

Dans le texte plus récent Fantasmagories (2005), c'est désormais la photographie qui est l'objet d'un tel soupçon critique (celui de ne pas être à même de témoigner du réel); plus succinctes mais analogues, d'autres remarques sur la « reproduction sonore » (la phonographie, préféronsnous dire) et (étonnamment) la peinture, s'y ajoutent. Rosset critique une « conception simpliste de la photographie » (F, p. 16), marquée par le « crédit extravagant» qu'une certaine doxa (le « on » du bon sens commun) accorde à ses images, du fait de l'automatisme - donc l'objectivité - de leur production. Avec la photographie, écrit Rosset, tout se passe comme si « on pouvait enfin voir le monde en personne, en direct, en vérité ». (F, p. 16)

On peut tout d'abord se demander si cette entreprise critique ne vient pas pour ainsi dire " après la bataille », et si, à l'aube du XXI siècle, le « crédit extravagant » accordé à la photographie n'a pas été remplacé par la croyance absolument inverse en la «manipulation » généralisée des esprits passifs par les images trompeuses. L'essentiel de la critique de Rosset porte sur une croyance humaine plutôt que sur le fait technique lui-même. Mais on ne saurait faire totalement l'économie de celui-ci. Que

5. Il semble apprécier les « trucages les plus cocasses » réalisés par des artistes doués du « sens du loufoque » $(\mathrm{F}, 16)$. 
signifie dans le cas de la photographie le fait de « représenter le réel »? Pour y répondre, Rosset en reste à un stade que l'on pourrait appeler, en reprenant les catégories sémiotiques de Peirce, le stade « iconique » : la représentation (son efficace à faire illusion et corollairement son échec à rendre compte du singulier) est fondée sur la notion de ressemblance avec son objet (le réel). Dans l'Objet singulier, Rosset écrit que le cinéma " suggère à s'y méprendre les traits de la vie réelle » c'est-à-dire « ses images, son mouvement, son bruit»: on peut s'étonner de cette expression « à s'y méprendre », qui dénote l'idée d'une illusion par forte ressemblance, comme si le cinéma, plutôt que de recueillir des empreintes matérielles du réel, en imitait ou simulait les percepts d'une manière quasi-hallucinatoire. En un certain sens, Rosset ignore volontairement ce qui fait la spécificité sémiotique des images obtenues par impression matérielle: leur statut d' « indices » selon la terminologie de Peirce, c'est-à-dire de signes « en connexion dynamique (y compris spatiale) et avec l'objet individuel d'une part et avec les sens ou la mémoire de la personne pour laquelle il sert de signe, d'autre part $»^{6}$. De ce point de vue, la photographie entre de plain-pied dans le « paradigme indiciaire », selon l'expression de l'historien Carlo Ginzburg, un paradigme que les études photographiques ont d'abord affirmé sans réserve ${ }^{7}$, puis nuancé ou critiqué $^{8}$. Chez Rosset le voilà - chose encore inédite - totalement ignoré.

Difficile pourtant d'en faire totalement l'économie, y compris lorsque ce que l'on vise est non la technique, mais les croyances que celle-ci suscite : ce paradigme, Rosset « tourne autour» sans jamais l'exposer de front. On en perçoit la prégnance lorsque se trouve abordée, s'agissant des photographies, la question de leur « conformité » au réel, une notion que Rosset alternativement admet et nie. Il l'admet au détour de quelques formules: lorsqu'il écrit que la photographie ne saurait " prétendre à plus qu'une conformité au réel » (F, p. 50), on peut comprendre que cette conformité est néanmoins possible, et qu'elle n'est peut-être pas si négligeable. Mais une telle possibilité se trouve ailleurs rejetée en des termes très explicites: « on ne peut jamais les prendre [les photographies]

6. Charles Sanders Peirce, Écrits sur le signe, traduits et présentés par G. Deladalle, Paris: Seuil, L'ordre philosophique, 1978, p. 158.

7. Dans le sillage des textes fondateurs : La Chambre claire de Roland Barthes et Le photographique. Pour une théorie des écarts de Rosalind Kraus.

8. Cf. Yves Michaud, «Critique de la crédulité. La logique de la relation entre l'image et la réalité », Études photographiques $\mathrm{n}^{\circ} 12,2002$, p. 111-125. 


\section{Photo-, phono- et cinématographie chez Clément Rosset}

pour des copies conformes de la réalité » (F, p. 35, nous soulignons). En un sens, Rosset admet l'indéniable: qu'il y a un lien matériel entre une photographie et son référent original. Mais il refuse d'en inférer une disposition incontestable de la photographie à se conformer au réel et à sa vérité.

Si elle porte sur une croyance diffuse et générale, la critique de Rosset n'en localise pas moins sa source : le célèbre ouvrage de Roland Barthes $L a$ Chambre claire (1980). Rosset résume la thèse de ce livre en la traduisant sous la forme d'une proposition apodictique: si photographie, alors réel. Barthes selon Rosset postulerait que « sera tenu pour réel tout ce dont on aura réussi à montrer une photographie », ce qui revient à dire que « la photographie est la seule garantie de l'existence du réel » (F, p. 19). La proposition logique est alors réfutée par le philosophe, sans que cette tâche ne soit trop ardue ${ }^{9}$; il suffit pour cela de dire deux choses : qu'il y a du réel hors de ce qui est photographié, et que ce qui est photographié et que l'on tient pour conforme au réel peut s'avérer illusoire, mensonger, irréel. Rosset estime pouvoir faire s'écrouler l'édifice théorique de $\mathrm{La}$ Chambre claire par l'argument, bien connu, de la falsification des images photographiques. À partir du moment où l'on convient qu'il est techniquement possible de truquer une photographie, de s'en servir comme « matériau brut à partir duquel il est loisible de travailler » (F, p. 29), il deviendrait impossible de considérer celle-ci comme la garantie fidèle de la réalité de ce que l'on voit, selon le noème du «ça-a-été », central dans l'ouvrage de Barthes.

C'est un argument très simple, en convient Rosset (qui raille au passage le «bon sens » de son aîné, F, p. 21). Et certes, nous ne dirons pas le contraire: une photographie truquée est, en un certain sens, mensongère sur le réel qu'elle restitue. Reste néanmoins à localiser le lieu du mensonge : qu'attend-on exactement que la photographie nous « révèle » d'un réel dont elle ne montre au mieux qu'une trace partielle et médiatisée, y compris lorsqu'elle est non truquée et « authentique »? De quelle réalité ou de quelle « vérité » la falsification nous détourne-t-elle?

9. On mesure particulièrement la rapidité de la lecture de Barthes par Rosset lorsque ce dernier, citant l'épisode du Lotus bleu où Tintin manque de se faire assassiner par un soi-disant photographe ayant dissimulé une mitrailleuse dans son appareil (F, p. 34), insiste sur le rapport de la photographie à la mort, sur la « mortification » que l'image opère sur son référent. Le philosophe semble oublier que c'est là précisément l'un des motifs majeurs de La Chambre claire, qui il est vrai omet de citer Tintin. 
Pour tenter de répondre à cette interrogation, il faut aller plus loin dans la caractérisation sémiotique de la photographie. La question de la vérité comme alternative binaire entre vrai et faux relève du troisième niveau de la taxinomie de Peirce, celui de l'interprétant - la « représentation mentale ${ }^{10}$ du signe qui opère la jonction entre celui-ci et son objet. À ce niveau sont distingués le signe rhématique - celui qui ne fait apparaître que la pure qualité de ce qu'il montre, «signe de possibilité qualitative $»^{11}$ - et le signe dicent ou dicisigne ${ }^{12}$ - celui qui est ou bien vrai ou bien faux, sans « fourni[r] de raison de sa vérité ou de sa fausseté $»^{13}$. Aussi un portrait est-il rhématique dès lors qu'il ne fait que montrer un individu (il ne saurait être faux ou vrai, à ce stade), il ne fait alors rien d'autre que « représente[r] toute une classe d'objets possibles: les personnes ressemblant à ce portrait $\rangle^{14}$. Il devient dicent, en revanche, lorsque par exemple une légende l'accompagne et l'indexe au réel. Si je vois un portrait du général de Gaulle au-dessous duquel est écrit le nom de Georges Pompidou, alors j'ai affaire à un signe dicent, car la mise en relation par contigüité de l'image et du texte crée un interprétant sous la forme de l'assertion - fausse, en l'occurrence -: «l'homme représenté ici est Georges Pompidou ».

Tournons-nous vers l'exemple que cite Rosset: les photographies du général de Gaulle prononçant son fameux « Vive le Québec libre » devant une place vide, que n'habitent que « de rares passants [...] occupés à lire leur journal » (F, p. 30). Il s'agit d'un cas typique de photomontage dissimulé, rassemblant dans une même image, avec une habileté pouvant faire illusion, deux réalités étrangères. Mais ce qui est faux n'est ni l'image du général de Gaulle, ni celle des passants indifférents (niveau rhématique): chacune de ces deux réalités aura dû, pour apparaître sur l'image, « se poser » à un moment donné devant un objectif. Ce qui est faux est non l'image mais l'interprétant que l'on produit spontanément,

10. Peirce, op. cit., p. 128.

11. Peirce, op. cit., p. 141.

12. Nous mettons délibérément de côté le signe argumental (« signe qui, pour son interprétant est un signe de loi », Peirce, op. cit., p. 141) car, selon la logique interne du système de Peirce, il ne peut relever que du symbole et non de l'indice (et encore moins de l'icône). Il nous semble difficile - mais la question pourrait être, ailleurs, posée - de rapporter l'image photographique au régime du symbole.

13. Peirce, op. cit., p. 167.

14. Nicole Everaert-Desmedt, «La sémiotique de Peirce », in Louis Hébert (dir.), Signo (en ligne), Rimouski (Québec), http://www.signosemio.com. 


\section{Photo-, phono- et cinématographie chez Clément Rosset}

mécaniquement ${ }^{15}$ à la vue d'une telle image truquée; ce qui est faux est l'énoncé déductif (niveau dicent) qui rapporterait la jonction artificielle des deux espaces-temps à la véracité d'un avoir-eu-lieu: « de Gaulle a prononcé son discours devant une place déserte ».

Réaffirmons donc le « ça-a-été » barthésien: il faudrait ne pas reconnaître à l'image le statut de photographie pour nier que la réalité (les réalités) que l'image désigne n'ai(en)t été, à un moment et à un lieu donnés, présentée(s) à un objectif, quelles qu'aient été les « manipulations » ultérieures. Dans les termes de Jean-Louis Déotte : « pour que cette image soit, il a bien fallu dans le passé qu'un objet réfléchisse un rayon lumineux et que ce dernier impressionne une pellicule photosensible, cela malgré les caviardages possibles, malgré les codes que décrivent les sémiologues de l'image $»^{16}$. Ce que Barthes nomme le nomos de l'appareil photographique, le " ça-a-été ", se situe à ce niveau seulement: «quelque chose s'est posé devant le petit trou ${ }^{17}$. Tout ce qui suit (le moment interprétatif) se dégage de cette loi et échappe ainsi à toute certitude. La manipulation peut à l'envi transformer, effacer le référent ou encore lui apposer une légende fallacieuse, une origine douteuse (telles les soi-disant photographies de "martiens, ovnis et autres soucoupes volantes », F, p. 18), le référent n'en restera pas moins ce qu'il est: cette « chose nécessairement réelle ${ }^{18}$ placée devant l'objectif.

Là où Rosset prend les termes " vérité » et « réalité » comme quasisynonymes, souvent accolés et interchangeables, nous pouvons proposer une distinction entre la vérité comme attribut vérifiable, validable, d'une assertion (c'est-à-dire comme objet d'un consensus) et la réalité comme lieu d'où les choses se donnent à percevoir, à saisir, à photographier. Le processus de production d'images photographiques n'aura jamais affaire qu'à cette dernière, ne saura rien saisir d'autre que ce que les rayons lumineux veulent bien lui fournir comme matière à impression. La question de la vérité, elle, ne se pose que quand l'image photographique s'articule à un contexte, génère des interprétants, des dicisignes.

15. L'aspect mécanique de l'indice, reposant sur un renvoi immédiat à l'objet, est souligné à maintes reprises par Peirce: «L'indice n'affirme rien; il dit seulement: "Là". il se saisit pour ainsi dire de vos yeux et les force à regarder un objet particulier et c'est tout » (Peirce, op. cit., p. 144).

16. Jean-Louis Déotte, Qu'est-ce qu'un appareil? Benjamin, Lyotard, Rancière, Paris : L'Harmattan, 2007 , p. 25.

17. Barthes, La Chambre claire, Paris: Gallimard/Les Cahiers du cinéma, 1980, p. 123.

18. Ibid., p. 120. 
Le court paragraphe de Fantasmagories consacré à la « reproduction sonore » confirme combien chez Rosset le sensible est ramené à des questions de l'ordre du discours (à commencer par le récurrent doute méthodique: ai-je affaire au réel/vrai ou à un simulacre?). Annoncé comme un prolongement d'idées déjà exposées au sujet de la photographie (« les techniques de reproduction sonore, nées plus tardivement encore que la photographie, sont sujettes au même soupçon d'infidélité au réel », F, p. 50), ce développement n'en cherche pas moins à dégager ce qui semble être une spécificité de la reproduction sonore par rapport à la photographie et la rend encore plus douteuse :

Pour en revenir au pouvoir de mensonge de la reproduction sonore, il est à remarquer que celui-ci outrepasse parfois les pouvoirs de la reproduction photographique puisqu'il est capable, non seulement de déformer un propos, mais encore de lui faire dire le contraire de ce qui a été dit. Prouesse dont la photographie est incapable: on ne voit pas bien, par exemple, en quoi pourrait consister une photographie représentant le « contraire » de la mère de Roland Barthes. (F, p. 53)

L'exemple de la parole n'est pas anodin: il ne concerne pas n'importe lequel des phénomènes sonores, mais celui qui passe par le langage. Réactivant la polysémie de la phoné grecque, tendue entre la dénotation de la voix humaine et le renvoi au pur son, entre le logos et le psophos, Rosset rabat ici le champ du sonore sur celui, bien différent, du verbal. La « prouesse » qu'il souligne est entièrement tributaire du langage et n'a rien à voir avec le sonore ou la phonographie; on pourrait de la même façon, avec autant d'embarras, se demander quel serait le " contraire " - notion éminemment linguistique, logique - d'enregistrements tel que celui du ressac maritime ou du trafic urbain, lesquels auraient dû être les objets de son enquête. Et c'est conformément à ce déplacement que, dans les lignes qui suivent, Rosset quitte sans plus d'explication le domaine du sonore au profit de celui de l'écriture, entrevoyant dans son « analogie » une vérité sur la manipulation sonore, alors qu'il n'est plus question que de textualité:

J'ai été moi-même victime d'un même détournement de ce que j'avais dit, ou plutôt écrit: un ouvrage, fait sans doute un peu vite, qui se proposait de faire le catalogue des imbécillités les plus flagrantes écrites par les 


\section{Photo-, phono- et cinématographie chez Clément Rosset}

philosophes contemporains, m'ayant attribué des phrases qui n'étaient pas de mon cru, et que j'avais citées moi-même comme exemple remarquable de sottise. Certes, il ne s'agit pas ici de reproduction sonore, mais de reproduction écrite. Cependant le procédé de falsification est analogue, ce qui montre qu'on peut truquer une reproduction de texte écrit aussi facilement qu'une reproduction de texte parlé. (F, p. 53-54)

Là où Rosset admettait que la technique photographique pouvait être renversée en « art capable de produire des images de plus ou moins grande valeur esthétique » $(\mathrm{F}, \mathrm{p} .35)$, il tend à ne considérer le procès de fixation du son que comme une simple duplication du réel en copie carbone: ersatz d'une parole dite, succédané d'un concert auquel on n'a pu assister, etc. La seule mention d'une alliance entre phonographie et production musicale se fait sur le mode d'une critique implicite (et semble-t-il peu informée) de la musique électroacoustique, assimilant tout travail sur du matériel phonographique à un simple bricolage: « le premier synthétiseur venu transforme à volonté le son d'une flûte en son de cor anglais, le barrissement d'un éléphant en un cri d'otarie, au point que ce jeu de possibilités infinies peut en arriver à dévoyer le talent du compositeur en le laissant s'abandonner à un pur jeu de timbre qui le dispense de composer » (F, p. 51). Ainsi, à la phonographie, Rosset dénie tout à la fois la possibilité de reproduire le phénomène sonore « vivant » et la puissance esthétique consistant à produire un sonore - une dimension du sonore - qui lui soit propre, qui puisse faire art, valoir comme musique, s'intégrer à la musique.

Ce double déni se traduit par l'emploi de l'expression peu heureuse de « reproduction sonore ». « Re-produire » le réel consisterait, à la lettre, plutôt que de dupliquer une matrice créée à partir de lui (le négatif de la photographie, par exemple), à reconduire les procès et les opérations qui l'ont fait venir à l'être. À créer un second réel, identique en tout point au premier car produit de la même façon. Dans Fantasmagories, Rosset va jusqu'à envisager parfois (ou plutôt prêter à la doxa qu'il attaque) cette perspective proprement impensable d'une image identifiée à son modèle :

Je ne mentionnerai ici que deux raisons principales qui interdisent, tant à la reproduction sonore qu'à la reproduction photographique, de prétendre à plus qu'une conformité au réel comparable, dans le meilleur des cas, aux photocopies dont un cachet et une signature officiels nous assurent 
qu'elles sont « conformes au document original », faute de pouvoir démontrer qu'elles lui sont identiques. (F, p. 50)

Rosset fait ici valoir la « conformité » à l'original comme continuité matérielle au détriment de l'identité comme continuité ontologique. Mais il n'est pas certain que la porte qu'il enfonce ne soit pas déjà largement ouverte. Comment en effet entendre rationnellement cette idée d'identité dans le cadre d'un rapport de représentation? Comment l'image pourrait-elle bien, aussi « réaliste » soit-elle, être identique à ce qu'elle représente? Certes, quelques expressions du langage courant désignent nonchalamment une image par la seule mention de son référent (« ceci est mon père, ceci est ma maison ", dit-on volontiers en montrant des photographies). Mais sauf cas exceptionnel de dérèglement de la raison, cet écart de langage n'amène nullement celui qui s'en rend fautif à prendre l'image d'une personne pour un « double de remplacement » (F, p. 73) de celle-ci.

Soucieux dans toute son œuvre de « démasquer les efforts, les extraordinaires gymnastiques intellectuelles auxquels s'adonnent la majorité des gens, et les philosophes en premier lieu, pour ne pas être en contact avec la réalité $\gg{ }^{19}$, Rosset ne considère le rapport aux images que selon cet angle, toujours le même depuis Le Réel et son double : l'éternelle illusion qui nous détourne d'un réel «tragique » que nous ne voulons pas voir. Mais par là - et sans doute tout à fait involontairement - il reconduit le topos postmoderne (ou plus précisément baudrillardien) selon lequel les images et représentations nous détournent du réel, voire même l'annihilent, le vident de sa substance ou de son authenticité. On connaît la thèse de Simulacres et Simulation de Jean Baudrillard (penseur pourtant très éloigné de Rosset). La profusion des images (des copies, des doubles, des clones) prive la réalité de son caractère unique, de sorte que le rapport entre l'original (le réel) et les copies (les images) se retourne: c'est le réel qui imite les images et non plus l'inverse. Ainsi Disneyland et Las Vegas auront-ils consacré le règne d'un monde où le modèle du clone, standardisé et « hyperréalisé », dicte au réel ses nouvelles architectures et ses modes de vie.

L'erreur la plus manifeste d'un tel raisonnement consiste à séparer et opposer radicalement le domaine du réel à celui de l'image,

19. Entretien accordé à Philosophie Magazine, art. cit., p. 58. 


\section{Photo-, phono- et cinématographie chez Clément Rosset}

au nom de la singularité du premier, et de l'infinie reproductibilité du second. Comme si notre appréhension du réel était immédiate et ne s'opérait pas, au contraire, à travers une multitude de médiations, parmi lesquelles les images, toutes les images. La seconde erreur consiste à rendre ces deux domaines incompatibles, comme si, par quelque système de vases communicants, l'essor de l'un impliquait le déclin de l'autre. Dans cette perspective, le réel est considéré comme une sorte de réservoir fini d'objets, fragilisé, précarisé ou mis en danger par la profusion des images; ou encore un ensemble clos dont la capacité d'inclusion est limitée et qui, passé un certain seuil, ne peut plus s'accommoder de ces multiples « augmentations » que sont les images.

Contre cette perspective, il convient assurément de rompre avec une certaine idée du réel, celle qui ne le concevrait qu'à travers ses « objets » (le titre L'Objet singulier le dit bien) en faisant l'impasse sur ses opérations productrices (ce par quoi quelque chose de réel advient). Le réel, nous le subissons certes (nous en éprouvons le tragique fatum sondé par Rosset dès son premier livre), mais nous le produisons également, par nos actes et nos désirs (on se souvient de la définition du désir comme « production de réel » selon Deleuze et Guattari). Le réel est certes cette extériorité hors de moi, mais il est également la perception singulière que j'en ai. Il n'est pas tant une collection d'objets à exemplaire unique que le terrain d'une expérience fait de moments absolument singuliers, située précisément entre l'extériorité de l'objet hors d'atteinte et l'intériorité pure, trompeuse et chimérique du double.

Cette redéfinition du réel n'est pas tant une négation des thèses de Rosset qu'une lecture sélective et critique de certains de ses postulats. En appendice de Le Réel, Traité de l'idiotie, Rosset écrit: « ne cherchez pas le réel ailleurs qu'ici et maintenant car il est ici et maintenant, seulement ici et maintenant » (TI, p. 152). Mais le chapitre introductif de L'Objet Singulier publié deux ans plus tard tient un tout autre discours, assurément plus pénétrant: en radicalisant les implications de la singularité du réel, il postule comme impossible toute appréhension du singulier qui ne passe pas par le truchement d'un double, toute considération du même qui ne s'effectue en référence à l'autre du même. Analysant la duplicité sémantique du concept même d'identité, qui désigne à la fois " ce qui est sans égal et ce qui est égal à quelque chose » (OS, p. 20), Rosset pose le double comme ce « quelque chose » qui « donne à penser » l'identité du réel, « invitant en quelque sorte au rêve de ce qu'elle est par la claire 
perception de tout ce qu'elle n'est pas » (OS, p. 21). Dès lors, tout en étant cette illusion qui détourne de l'appréhension immédiate du réel, le double est également le moyen paradoxal de sa saisie en négatif, en creux. Si l'ontologie du réel entreprise par Rosset est une « ontologie négative » (OS, p. 28), c'est qu'elle doit passer par l'examen critique de ces doubles et représentations, à défaut de pouvoir appréhender la singularité même.

Nous pouvons suivre Rosset sur l'idée que notre appréhension du réel est sans cesse médiatisée, c'est-à-dire qu'elle s'opère par le truchement de représentations. Nous pouvons en revanche nous en désolidariser lorsqu'il assimile ces représentations à des " doubles » ou des " chimères », plutôt que des « voies d'accès au réel » (TI, p. 45), voire des éléments du réel même - car participant de l'expérience, absolument singulière, que nous en faisons. Rosset lui-même va dans cette direction lorsque, dans le petit texte Impressions fugitives (2004), il se propose de définir un double émancipé de l'idée d'illusion: non plus négatif et illusoire, mais positif et nécessaire. Il s'agit du double « de proximité », représenté par trois objets: le reflet, l'ombre et l'écho.

Le double est sans doute le symptôme majeur du refus du réel et le facteur principal de l'illusion; mais il existe certains doubles qui sont au contraire des signatures du réel garantissant son authenticité: telle précisément l'ombre qui vient à manquer à la femme sans ombre, tels aussi le reflet et l'écho. (IF, p. 9)

Moment de renversement radical dans la pensée de Rosset, puisque cette " positivation » du double lui attribue une qualité qui est précisément celle qu'il lui dénie dans les écrits antérieurs depuis Le Réel et son double (et jusqu'au pourtant postérieur Fantasmagories) : non seulement la garantie d'authenticité, exprimée par l'indice de la « signature », mais surtout la solidarité avec le réel. Ce ne sont pas « des prolongements fantomatiques du réel, mais des compléments nécessaires qui sont ses attributs obligés » (IF, p. 10). Ce double n'a dès lors plus aucune raison de s'opposer au réel, voire de s'en distinguer. Pourquoi limiter arbitrairement cette notion de double « positif » à ces trois éléments? Pourquoi ne pas y inclure les images et représentations qui participent de notre appréhension du réel plutôt qu'elles ne nous en détournent? Rosset s'approche de cette idée dans l'avant-propos de Fantasmagories lorsque, dépouillant l'image 
de toute référence au double trompeur, il écrit - assurément la phrase la plus lucide de tout l'ouvrage - que la photographie, la « reproduction sonore » et la peinture (mais on pourrait bien entendu élargir cette liste) sont à considérer comme « des réalités à part entière qu'il serait par conséquent vain de distinguer de la réalité en général dont elles partagent tous les privilèges » $(\mathrm{F}, \mathrm{p} .11)$. 